\title{
AN ISOPERIMETRIC THEOREM ON THE CUBE AND THE KINTCHINE-KAHANE INEQUALITIES
}

\author{
MICHEL TALAGRAND
}

(Communicated by W. J. Davis)

\begin{abstract}
For vectors $x_{1}, \ldots, x_{n}$ in a Banach space, we bound the deviation of $\left\|\sum_{i \leq n} \varepsilon_{i} x_{i}\right\|$ from its median.
\end{abstract}

1. Results. Consider the set $\{-1,1\}^{n}$, provided with its canonical probability measure $P_{n}$ that gives mass $2^{-n}$ to each point. We will consider $\{-1,1\}^{n}$ as a subset of the $n$-dimensional Hilbert space $\mathbf{R}^{n}$. For a nonempty subset of $A$ of $\{-1,1\}^{n}$, we set $\phi_{A}(x)=\inf \left\{\|x-y\|_{2} ; y \in \operatorname{conv} A\right\}$.

THEOREM 1. $E \exp \left(\phi_{A}^{2} / 8\right) \leq 1 / P_{n}(A)$.

From Chebyshev's inequality we get

COROLlaRY 2. For all $t \geq 0$, we have $P_{n}\left(\left\{\phi_{A} \geq t\right\}\right) \leq\left(1 / P_{n}(A)\right) e^{-t^{2} / 8}$.

To understand this result, it might be helpful to compare it with classical results concerning the Hamming distance. The Hamming distance $d(s, t)$ between $s, t \in$ $\{0,1\}^{n}$ is the number of coordinates where $s$ and $t$ differ. For a subset $A$ of $\{-1,1\}^{n}$, we set $d_{A}(x)=\inf \{d(x, y) ; y \in A\}$. The largest possible value of $P_{n}\left(\left\{d_{A} \geq t\right\}\right)$ when $P_{n}(A)$ is given is known. The sets for which $P_{n}\left(\left\{d_{A} \geq t\right\}\right)$ is maximum are identified in [3]. In particular, when $P_{n}(A)=1 / 2$, it is known (see [1]) that

$$
P_{n}\left(\left\{d_{A} \geq t \sqrt{n}\right\}\right) \leq \frac{1}{2} e^{-2 t^{2}} .
$$

To make the connection with Corollary 2 , we observe the following.

Fact. $2 d_{A} \leq \sqrt{n} \phi_{A}$.

PROOF. Fix $x$, and consider the linear functional $\theta$ on $\mathbf{R}^{n}$ given by

$$
\theta(z)=\sum_{i \leq n} n^{-1 / 2} x_{i} z_{i}
$$

It is of norm 1. For any $y \in A$, we have

$$
\theta(x-y)=\sum_{i \leq n} \frac{1}{\sqrt{n}} x_{i}\left(x_{i}-y_{i}\right)=\frac{2}{\sqrt{n}} d(x, y) \geq \frac{2}{\sqrt{n}} d_{A}(x) .
$$

It follows that for each $y \in \operatorname{conv} A$, we have $\theta(x-y) \geq(2 / \sqrt{n}) d_{A}(x)$ so that $\|x-y\|_{2} \geq(2 / \sqrt{n}) d_{A}(x)$, which proves the fact.

Received by the editors September 23, 1987 and, in revised form, January 10, 1988.

1980 Mathematics Subject Classification (1985 Revision). Primary 60E15, 60B11; Secondary 05 C35.

Key words and phrases. Hamming distance, concentration of measure, Kintchine-Kahane inequalities, Bernoulli randomization.

This research was partially supported by NSF Grant DMS-860 3951. 
The fact shows that

$$
\left\{d_{A} \geq(t / 2) \sqrt{n}\right\} \subset\left\{\phi_{A} \geq t\right\} .
$$

While (1-1) provides the estimate

$$
P_{n}\left(\left\{d_{A} \geq(t / 2) \sqrt{n}\right\}\right) \leq \frac{1}{2} e^{-t^{2} / 2},
$$

Corollary 2 provides a larger bound for a larger set:

$$
P_{n}\left(\left\{\phi_{A} \geq t\right\}\right) \leq 2 e^{-t^{2} / 8} .
$$

Our method of proof cannot give in Theorem 1 a coefficient better than $1 / 8$. It is to be noted however that (1-3) is better than the estimates for $P_{n}\left(\left\{d_{A} \geq t \sqrt{n} / 2\right\}\right)$ that one obtains by martingale methods (see [4, p. 36]). We do not know the answer to the following.

Problem. What is the value of

$$
\max \left\{P_{n}\left(\left\{\phi_{A}(x) \geq t\right\}\right) ; P_{n}(A)=a\right\} ?
$$

This, and a related problem, is discussed in $\S 3$.

THEOREM 3. Consider a convex Lipschitz function $f$ on $\left(\mathbf{R}^{n},\|\cdot\|_{2}\right)$, of Lipschitz constant $\sigma$. Denote by $M$ a median of $f\left(\right.$ for $\left.P_{n}\right)$. Then we have for all $t \geq 0$,

$$
P_{n}(\{|f-M|>t\}) \leq 4 e^{-t^{2} / 8 \sigma^{2}} .
$$

An interesting feature of this result is that it is really specific to convex functions. To see this, let $A=\left\{x \in\{-1,1\}^{n}: \sum_{i \leq n} x_{i} \leq 0\right\}$ and define $f(x)=$ $\inf \left\{\|x-y\|_{2}: y \in A\right\}$, so that $f$ has a Lipschitz constant 1 , and $M=0$. It is easy to see that for $y \in\{-1,1\}^{n}$, we have $f(y) / \sqrt{2}=d(y, A)^{1 / 2}=\left(\left(\sum_{i \leq n} y_{i}\right)^{+}\right)^{1 / 2}$. It follows from the central limit theorem that $P_{n}\left(\left\{f>c n^{1 / 4}\right\}\right) \geq 1 / 4$ for some constant $c$ independent of $n$.

Corollary 4. Consider a Banach space $E$, and $\left(x_{i}\right)_{i \leq n}$ in $E$. Let

$$
\sigma^{2}=\sup \left\{\sum_{i \leq n} x^{*}\left(x_{i}\right)^{2} ; x^{*} \in E^{*},\left\|x^{*}\right\| \leq 1\right\} .
$$

Consider an i.i.d. sequence $\left(\varepsilon_{i}\right)_{i \leq n}$ of Bernoulli random variables. Let $M$ be a median of $\left\|\sum_{i \leq n} \varepsilon_{i} x_{i}\right\|$. Then for all $t \geq 0$,

$$
P\left(\left\{||\left|\sum_{i \leq n} \varepsilon_{i} x_{i} \|-M\right| \geq t\right\}\right) \leq 4 e^{-t^{2} / 8 \sigma^{2}} .
$$

This inequality should be compared with the inequality

$$
P\left(\left\{||\left|\sum_{i \leq n} g_{i} x_{i}\right||-M| \geq t\right\}\right) \leq \frac{2}{\sigma \sqrt{2 \pi}} \int_{t}^{\infty} e^{-u^{2} / 2 \sigma^{2}} d u \leq 2 e^{-t^{2} / 2 \sigma^{2}},
$$

where the second inequality holds for $t \geq \sigma(2 \pi)^{1 / 2}$, and where $\left(g_{i}\right)_{i \leq n}$ are i.i.d. $N(0,1)$ and $M$ is now the median of $\left\|\sum_{i \leq n} g_{i} x_{i}\right\|$. (Inequality (1-6) is a well-known consequence of Borell's isoperimetric inequality [2].) Inequality (1-5) allows one to 
simplify (at least conceptually) the theory of probability in a Banach space, as it often allows one to replace Gaussian randomization by Bernoulli randomization.

By a standard computation, we deduce from Corollary 4 the following version of the Kintchine-Kahane inequalities.

COROLLARY 5. There exists a universal constant $K$, such that for any elements $x_{1}, \ldots, x_{n}$ of a Banach space, we have, for $p \geq 1$

$$
\left\|\sum_{i \leq n} \varepsilon_{i} x_{i}\right\|_{p} \leq\left\|\sum_{i \leq n} \varepsilon_{i} x_{i}\right\|_{1}+K \sigma p^{1 / 2}
$$

where $\sigma$ is given by $(1-4)$.

\section{Proofs.}

Proof of THEOREM 1 We first consider the case where $\operatorname{card} A=1$. Then

$$
E \exp \left(\frac{\phi_{A}^{2}}{8}\right)=2^{-n} \sum_{0 \leq i \leq n}\left(\begin{array}{c}
n \\
i
\end{array}\right) e^{i / 2}=\left(\frac{1+e^{1 / 2}}{2}\right)^{n} \leq 2^{n}=\frac{1}{P_{n}(A)}
$$

since $e^{1 / 2}<e<3$.

Next, we prove Theorem 1 when $n=1$. It remains only to consider the case where $A=\{-1,1\}$, so that $\phi_{A}(x) \equiv 0$, and the result holds.

We now prove Theorem 1 by induction over $n$. Assuming it holds for $n$, we prove it for $n+1$. It is enough to consider the case where $A$ has at least 2 points. Identifying $\{-1,1\}^{n+1}$ with $\{-1,1\}^{n} \times\{-1,1\}$, we can suppose that $A=A_{-1} \times$ $\{-1\} \cup A_{1} \times\{1\}$ where $A_{-1}, A_{1} \neq \varnothing, A_{-1}, A_{1} \subset\{-1,1\}^{n}$. For definiteness we assume that $P_{n}\left(A_{-1}\right) \leq P_{n}\left(A_{1}\right)$. We observe that for $x \in\{-1,1\}^{n}$, we have

$$
\phi_{A}((x, 1)) \leq \phi_{A_{1}}(x) .
$$

Fact. For $x \in\{-1,1\}^{n}$, and $0 \leq \alpha \leq 1$, we have

$$
\phi_{A}^{2}((x,-1)) \leq 4 \alpha^{2}+\alpha \phi_{A_{1}}^{2}(x)+(1-\alpha) \phi_{A_{-1}}^{2}(x) .
$$

PROOF OF FACT. For $i=-1,1$, let $z_{i} \in \operatorname{conv} A_{i}$ such that $\left\|x-z_{i}\right\|_{2}=\phi_{A_{i}}(x)$. We observe that $\left(z_{i}, i\right) \in \operatorname{conv} A$, so that $z=\left(\alpha z_{1}+(1-\alpha) z_{-1},-1+2 \alpha\right) \in \operatorname{conv} A$. Now

$$
\begin{aligned}
\|(x,-1)-z\|_{2}^{2} & =4 \alpha^{2}+\left\|x-\left(\alpha z_{1}+(1-\alpha) z_{-1}\right)\right\|_{2}^{2} \\
& =4 \alpha^{2}+\left\|\alpha\left(x-z_{1}\right)+(1-\alpha)\left(x-z_{-1}\right)\right\|_{2}^{2} \\
& \leq 4 \alpha^{2}+\alpha\left\|x-z_{1}\right\|_{2}^{2}+(1-\alpha)\left\|x-z_{-1}\right\|_{2}^{2}
\end{aligned}
$$

by the triangle inequality and the convexity of $t^{2}$. This proves the fact.

For $i=-1,1$, we set $u_{i}=E \exp \left(\phi_{A_{i}} / 8\right)$, and $v_{i}=1 / P\left(A_{i}\right)$, so that $u_{i} \leq v_{i}$ by the induction hypothesis. From (2-1), (2-2), we have for all $0 \leq \alpha \leq 1$,

$$
\begin{aligned}
E \exp \left(\phi_{A}^{2} / 8\right) \leq & \frac{1}{2} E \exp \left(\phi_{A_{1}}^{2} / 8\right) \\
& +\frac{1}{2} E \exp \left(\alpha^{2} / 2+\alpha \phi_{A_{1}}^{2} / 8+(1-\alpha) \phi_{A_{-1}}^{2} / 8\right) \\
\leq & \frac{1}{2} u_{1}+\frac{1}{2} e^{\alpha^{2} / 2} u_{1}^{\alpha} u_{-1}^{1-\alpha} \\
\leq & \frac{1}{2} v_{1}\left[1+e^{\alpha^{2} / 2}\left(v_{1} / v_{-1}\right)^{\alpha-1}\right]
\end{aligned}
$$


by Hölder's inequality and since $u_{i} \leq v_{i}$. The value of $\alpha$ that minimizes the above expression is $\alpha=-\log \left(v_{1} / v_{-1}\right)$, but, in order not to have to consider the case where $\alpha \geq 1$, we take $\alpha=1-v_{1} / v_{-1}$, which gives

$$
E \exp \left(\phi_{A}^{2} / 8\right) \leq \frac{1}{2} v_{1}\left[1+e^{\alpha^{2} / 2}(1-\alpha)^{\alpha-1}\right]
$$

LEMMA. If $0 \leq \alpha<1$, we have

$$
1+e^{\alpha^{2} / 2}(1-\alpha)^{\alpha-1} \leq 4 /(2-\alpha) .
$$

Indeed, this is equivalent to saying that

$$
e^{\alpha^{2} / 2}(1-\alpha)^{\alpha-1} \leq(2+\alpha) /(2-\alpha)
$$

or that

$$
\alpha^{2} / 2-(1-\alpha) \log (1-\alpha) \leq \log (1+\alpha / 2)-\log (1-\alpha / 2) .
$$

But this is easily seen by consecutive differentation.

We now have

$$
\begin{aligned}
E \exp \left(\phi_{a}^{2} / 8\right) & \leq \frac{1}{2} v_{1}\left(\frac{4}{2-\alpha}\right)=v_{1}\left(\frac{2}{1+v_{1} / v_{-1}}\right)=\frac{2}{1 / v_{1}+1 / v_{-1}} \\
& =\frac{2}{P_{n}\left(A_{1}\right)+P_{n}\left(A_{-1}\right)}=\frac{1}{P_{n+1}(A)} .
\end{aligned}
$$

This finishes the proof of Theorem 2.

PROOF OF THEOREM 3. By definition of a median, we have

$$
P_{n}(\{f \geq M\}) \geq 1 / 2, \quad P_{n}(\{f \leq M\}) \geq 1 / 2 .
$$

Let $A=\{f \leq M\}$. Since $f$ is convex, we have $f \leq M$ on conv $A$. Since $f$ has a Lipschitz constant $\sigma$, we have $f(x) \geq M+t \Rightarrow \phi_{A}(x) \geq t / \sigma$. Hence, by Corollary 2 ,

$$
P_{n}(\{f \geq M+t\}) \leq P_{n}\left(\left\{\phi_{A} \geq t / \sigma\right\}\right) \leq 2 e^{-t^{2} / 8 \sigma^{2}} .
$$

Now let $t>0$, and consider the set $B=\{f \leq M-t\}$. Let $u<t$. We see as before that

$$
f(x) \geq M-t+u \Rightarrow \phi_{B}(x) \geq u / \sigma
$$

so, by Corollary 2, we have

$$
1 / 2 \leq P_{n}(\{f \geq M-t+u\}) \leq\left(1 / P_{n}(B)\right) e^{-u^{2} / 8 \sigma^{2}}
$$

and $P_{n}(B) \leq 2 e^{-u^{2} / 8 \sigma^{2}}$. Letting $u \rightarrow t$, we have $P_{n}(B) \leq 2 e^{-t^{2} / 8 \sigma^{2}}$. This proves Theorem 3.

To prove Corollary 4, we apply Theorem 3 to the function $f: u \rightarrow\left\|\sum_{i \leq n} u_{i} x_{i}\right\|$ on $\mathbf{R}^{n}$. This function is obviously convex. Now, for $u, v \in \mathbf{R}^{n}, x^{*} \in E^{*},\left\|x^{*}\right\| \leq 1$, we have

$$
\begin{aligned}
\left|x^{*}\left(\sum_{i \leq n} u_{i} x_{i}\right)-x^{*}\left(\sum_{i \leq n} v_{i} x_{i}\right)\right| & \leq\left|\sum_{i \leq n}\left(u_{i}-v_{i}\right) x^{*}\left(x_{i}\right)\right| \\
& \leq\left(\sum_{i \leq n} x^{*}\left(x_{i}\right)^{2}\right)^{1 / 2}\|u-v\|_{2}
\end{aligned}
$$

so that $|f(u)-f(v)| \leq \sigma\|u-v\|_{2}$ and $f$ has a Lipschitz constant $\sigma$. 
3. Some problems of combinatorics. The first problem is, as mentioned in the introduction, the determination of the function

$$
h(a, t)=\max \left\{P_{n}\left(\left\{\phi_{A} \geq t\right\}\right) ; P_{n}(A)=a\right\} .
$$

This problem seems difficult. The reason is that the extremal sets (i.e. the sets for which $\left.P_{n}\left(\left\{\phi_{A} \geq t\right\}\right)=h(a, t), P_{n}(A)=a\right)$ depend on $t$. To see it, we note that for $a=1 / 2$, the set $A=\left\{x ; x_{1}=-1\right\}$ is extremal for $t<2$ (since $P_{n}(\{\phi(x, A) \geq t\})=$ $1 / 2$ is as large as possible) but is not extremal for $t>2$. Another related problem is as follows. Suppose that we are given a set $A \subset\{-1,1\}^{n}$, and for each $x \in A$ an element $f_{x}=\left(f_{x, i}\right)_{i \leq n}$ of $\mathbf{R}^{n}$, of norm one and of positive components. We can consider the set

$$
B=\left\{y \in\{-1,1\}^{n}, \forall x \in A, \sum_{i \leq n}\left|y_{i}-x_{i}\right| f_{x, i}>t\right\} .
$$

Knowing $P_{n}(A)$, how large can $P_{n}(B)$ be? We note that in the case where $f_{x, i}=$ $n^{-1 / 2}$ for all $x \in A$, all $i \leq n$, then $B=\{y: d(x, A)>t \sqrt{n} / 2\}$ and the answer is known in that case. In the general case, Corollary 2 implies the bound $P_{n}(B) \leq$ $\left(1 / P_{n}(A)\right) e^{-t^{2} / 8}$. Indeed, since $\left|y_{i}-x_{i}\right|=x_{i}\left(x_{i}-y_{i}\right)$, we have $\phi_{B}(x) \geq t$ on $A$, so by Corollary 2, we have $P_{n}(A) \leq\left(1 / P_{n}(B)\right) e^{-t^{2} / 8}$.

ACKNOWLEDGMENTS. I am indebted to N. Alon for useful comments, and to S. Szarek for polishing the proof of Theorem 1, and in particular for obtaining the coefficient $1 / 8$, which is the best possible by this method of proof.

\section{REFERENCES}

1. D. Amir and V. Milman, Unconditional symmetric sets in n-dimensional normed spaces, Israel J. Math. 37 (1980), 3-20.

2. C. Borell, The Brunn-Minkowski inequality in Gauss space, Invent. Math. 30 (1975), 205-216.

3. L. H. Harper, Optimal numbering and isoperimetric problems on graphs, J. Combin. Theory 1, (1966), 385-393.

4. V. Milman and G. Schechtman, Asymptotic theory of finite dimensional normed spaces, Lecture Notes in Math., vol. 1200, Springer-Verlag, New York and Berlin, 1986.

Equipe d'ANalyse-Tour 46, Université Paris Vi, 4 Place Jussieu, 75230 Paris, FRANCE

Department of Mathematics, Ohio State University, Columbus, Ohio 43210 